\title{
DIAGNÓSTICO DEL CLIMA LABORAL ENTRE ADMINISTRATIVOS NOMBRADOS Y CONTRATADOS DE LA FACULTAD DE CIENCIAS ADMINISTRATIVAS DE LA UNIVERSIDAD NACIONAL MAYOR DE SAN MARCOS
}

\author{
Ebor Fairlie Frisancho* \\ efairlie@unmsm.edu.pe
}

\begin{abstract}
RESUMEN
La preocupación por optimizar la productividad en las organizaciones y la creciente valoración del sistema humano social como el capital más importante para el éxito organizacional determina la demanda por servicios que permitan conocer el funcionamiento de procesos y resultados asociados al desempeño laboral.

Se aplicó una encuesta a los trabajadores administrativos de la Facultad de Ciencias Administrativas de la Universidad Nacional Mayor de San Marcos (UNMSM), que fue evaluada por el software Clima Laboral CL-SPC que analiza cinco factores: autorealización, involucramiento laboral, supervisión, comunicación y condiciones laborales.

Los resultados, en general, reflejan que el factor de autorrealización no está completamente desarrollado entre los trabajadores administrativos, quienes no se sienten identificados con la gestión (políticas), mientras que la comunicación laboral denota que son trasladados a otros puestos en poco tiempo y que las condiciones laborales estuvieron fijas hasta el 2010.

Esta encuesta se tomó cuando el Dr. Roger Loayza aún se encontraba como decano, pero es importante considerar que también influyen en el resultado obtenido las políticas de la gestión.
\end{abstract}

Palabras claves: Clima laboral, CL-SPC, personal administrativo, universidad.

\section{ABSTRACT}

Concern for optimizing productivity in organizations and the increasing social value to the human system as the most important capital for organizational success determines the demand for services that are aware of the demand for services that allow the functioning of processes and outcomes related to job performance .

A survey of office workers Faculty of Administrative Sciences of the Universidad Nacional Mayor de San Marcos and evaluated by the CL-WORK CLIMATE software that analyzes SPC 5 factors of self, JOB INVOLVEMENT, monitoring, communication and working conditions.

\footnotetext{
* Doctor en Administración. Magister en Administración. Licenciado en Administración de Empresas. Profesor auxiliar e investigador del Instituto de Investigaciones de la Facultad de Ciencias Administrativas. Coordinador Académico de la Unidad de Posgrado. Asesor de la Oficina de Calidad Académica y Acreditación de la Facultad de Ciencias Administrativas (UNMSM).
} 
The overall results show that the factor of self-realization is not complete, do not identify with management (policies), denotes labor and communication points are changed in a short time and working conditions fixed only until 2010.

This survey was taken when he was still Dr. Roger Loayza as Dean and also influence management policies

Keywords: Employee Satisfaction, CL-SPC, staff, University

\section{INTRODUCCIÓN}

Las exigencias del mundo contemporáneo obligan a la Facultad de Ciencias Administrativas de la Univeridad Nacional Mayor de San Marcos (UNMSM) al desarrollo de tendencias en las organizaciones centradas en la maximización de los sistemas tecnológicos de productividad y, fundamentalmente, del sistema humano y es aquí que ante esa necesidad de evaluar este desarrollo se hace necesario realizar un diagnóstico para determinar nuevos esquemas de interacción para encarar tanto la cultura y el entorno, asi como la productividad y calidad de nuestra Facultad.

$\mathrm{Al}$ usar esta herramienta para medir la escala del clima laboral en nuestra facultad, se evalúa la autorealización, involucramiento laboral, supervisión, comunicación y condiciones laborales de los trabajadores administrativos. Los resultados son mas que sorprendentes y reflejan el estado del personal administrativo para estos factores.

Este diagnóstico servirá para que en la próxima gestión se evalúe la importancia de los factores de interacción relevantes para plantear soluciones acertadas, que permitan mejorar la calidad de vida laboral de los trabajadores de la facultad.

\section{MARCO TEÓRICO}

La Escala CL-SPC fue diseñada y elaborada por la psicóloga Sonia Palma, profesora investigadora de la Universidad Ricardo Palma.

El instrumento fue diseñado con la ténica de Likert, y comprende una versión final de 50 ítems que exploran el clima laboral viendo dos variables: la percepción del trabajador con respecto a su ambiente laboral, y en función a posibilidades de realización personal, involucramiento con la tarea asignada, supervisión que recibe, acceso a la información relacionada con su trabajo en coordinación con sus demás compañeros y condiciones laborales que facilitan su tarea.

La aplicación del instrumento mide cinco factores:
- Autorealización.

- Involucramiento laboral.

- Supervisión.

- Comunicación.

- Condiciones laborales.

La aplicación del instrumento será computarizada para luego sacar estadísticas y hacer comparaciones con las variables de estudio.

Los trabajos encontrados anteriormente solo detallan estudios del personal administrativo por otros instrumentos, inclusive hechos por el Dr. Alejandro Loli Pineda, aplicados al personal nombrado de la Universidad Nacional Mayor de San Marcos, mas no al personal CAS o contratado que en nuestra Facultad son casi seis veces más trabajadores que el personal administrativo nombrado.

\section{La importancia de la satisfacción laboral}

El tema de la satisfacción laboral es de gran interés porque nos indica la habilidad de la organización para satisfacer las necesidades de los trabajadores; además, porque existen muchas evidencias de que los trabajadores insatisfechos faltan a trabajar con más frecuencia y suelen renunciar más. En contraparte, se ha demostrado que los empleados satisfechos gozan de mejor salud y viven más años.

Además, la satisfacción laboral se refleja en la vida particular del empleado y es de gran interés "en los últimos años por constituirse en resultados organizacionales que expresan el grado de eficacia, eficiencia y efectividad alcanzado por la institución; como tal, son indicadores del comportamiento de los que pueden derivar políticas y decisiones institucionales" (Palma, 1999, p. 24).

Es más probable que los empleados satisfechos sean ciudadanos satisfechos, ya que adoptarán una actitud más positiva ante la vida en general y representarán, para la sociedad, personas más sanas, en términos psicológicos.

Actualmente se presta mayor interés a la 
calidad de vida laboral a diferencia de años anteriores en donde se buscaba la relación con el rendimiento. "[...] subyace la idea de que las personas trabajen bien, pero sintiéndose bien; o a la inversa, que estén a gusto en el trabajo, al tiempo que ofrecen un resultado satisfactorio" (Peiró, 1996, p. 344).

Es un hecho observable que los trabajadores que se sienten satisfechos en su trabajo, sea porque se consideran bien pagados o bien tratados, sea porque ascienden o aprenden, son quienes producen y rinden más. A la inversa, los trabajadores que se sienten mal pagados, mal tratados, atascados en tareas monótonas, sin posibilidades de ampliar horizontes de comprensión de su labor, son los que rinden menos, es decir, son los más improductivos.

"Todos somos capaces de percibir claramente lo benéfico, agradable y estimulante de estar en el trabajo con un grupo de personas que se llevan bien, que se comprenden, que se comunican, que se respetan, trabajan en armonía y cooperación. La buena atmósfera en el trato es indispensable para lograr un elevado rendimiento individual y colectivo de un grupo humano de trabajo, como lo es el oxígeno para el normal funcionamiento de los pulmones y la respiración, lo cual se logra más que nada por una labor conciente de los jefes". (Atalaya, 1999, p. 50).

Cuadro 1. Breve reseña de las facetas de la satisfacción Laboral según algunos autores

\begin{tabular}{|c|c|c|c|c|}
\hline BEER (1964) & LOCKE (1976) & $\begin{array}{c}\text { GRAFFIN Y BATEMAN } \\
(1986)\end{array}$ & JHONS (1988) & PALMA (1999) \\
\hline Compañía & $\begin{array}{c}\text { Eventos o condiciones de } \\
\text { trabajo }\end{array}$ & Trabajo & Reconocimiento & $\begin{array}{l}\text { Condiciones } \\
\text { físicas }\end{array}$ \\
\hline Trabajo mismo & Salario & Paga & Beneficios & Remuneración \\
\hline Compañeros & Promoción & Supervisión & $\begin{array}{l}\text { Condiciones de } \\
\text { trabajo }\end{array}$ & $\begin{array}{c}\text { Políticas } \\
\text { administrativas }\end{array}$ \\
\hline Objeto del contexto & Reconocimiento & Beneficios & Supervisión & $\begin{array}{c}\text { Relaciones } \\
\text { sociales }\end{array}$ \\
\hline \multirow{4}{*}{ De trabajo } & \multirow{2}{*}{ Beneficios } & Oportunidades de promoción & $\begin{array}{c}\text { Compañeros de } \\
\text { trabajo. }\end{array}$ & $\begin{array}{l}\text { Desarrollo } \\
\text { personal }\end{array}$ \\
\hline & & Condiciones de trabajo & \multirow[b]{3}{*}{$\begin{array}{c}\text { Políticas de } \\
\text { empresa }\end{array}$} & $\begin{array}{l}\text { Desempeño de } \\
\text { tareas }\end{array}$ \\
\hline & \multirow{2}{*}{$\begin{array}{l}\text { Agentes de satisfacción: } \\
\text { Supervisión } \\
\text { Compañeros } \\
\text { Compañía } \\
\text { Dirección }\end{array}$} & Compañeros & & \multirow[b]{2}{*}{$\begin{array}{c}\text { Relación con la } \\
\text { autoridad }\end{array}$} \\
\hline & & Prácticas de la organización & & \\
\hline
\end{tabular}

Fuente: Elaboración Propia

\section{Tendencias generales de satisfacción}

El grado de satisfacción o insatisfacción varía mucho de persona a persona. Sin embargo, hay ciertas tendencias generales observadas en la población.

Halloran y Bentosn (1987) encontraron que existe una correlación positiva entre la satisfacción laboral y las variables edad, género, experien- cia laboral y nivel ocupacional. Los determinantes más importantes de la satisfacción laboral son:

- Edad. La razón por la cual la edad está asociada al nivel de satisfacción es en gran parte desconocida. Cuando la persona ingresa a la vida laboral su trabajo es algo nuevo e interesante, y las exigencias de la organización son aún muy bajas, lo cual produce un buen des- 
empeño y, en consecuencia, un alto nivel de satisfacción. A medida que avanza en edad, el trabajo se vuelve más rutinario y las exigencias mayores, con lo cual decrece el desempeño y el nivel de satisfacción. A partir de los 30 años la persona tiene la oportunidad de realizar tareas más ricas, lograr mejores niveles de desempeño y un mayor nivel de satisfacción laboral. Hacia los 60, la persona experimenta un declive de sus capacidades y un horizonte más corto de actuación, lo que origina un menor esfuerzo al ejecutar el trabajo. Así, su desempeño decae y la satisfacción baja.

- Género. En investigaciones realizadas en Estados Unidos se ha demostrado que las mujeres se sienten contentas con su trabajo y otras demuestran lo contrario. Algunos resultados de la investigación indican que están más insatisfechas que los varones. Se sabe que les interesan aspectos del trabajo por los que el hombre no muestra ningún interés en absoluto. Por ejemplo, las mujeres con desventajas necesitaban más sentir gusto por su trabajo y tener un buen jefe; en cambio, los hombres con desventajas se preocupaban más por la oportunidad de demostrar su utilidad y contar con un trabajo seguro. Por otra parte, ellas sentían más interés por la calidad de las relaciones interpersonales y las condiciones de trabajo.

Es natural que haya distintas fuentes de satisfacción para quienes escogen una carrera en el mundo de los negocios y las que trabajan a fin de contribuir al ingreso familiar. Las motivaciones y satisfacciones de las mujeres profesionales se parecen más a los de los ejecutivos. Ellas obtienen más satisfacción con su trabajo en cambio las madres no lo consideran el principal centro de su vida, pues se sienten realizadas en su papel de madre, esposa y ama de casa, (Schultz, 1998, p. 264).

Palma (1999), en un reciente estudio realizado en la ciudad de Lima Metropolitana, Perú, encontró que las diferencias por género favorecen a la mujer quien tiene un mejor nivel de satisfacción laboral.

- Experiencia laboral. La misma explicación se aplica para entender la asociación entre la experiencia dentro de la empresa y el nivel de satisfacción logrado. Cuando la persona empieza a realizar cualquier trabajo le ocurre lo mismo que a un joven recién iniciado en la vida laboral: al comienzo todo le parece nuevo e interesante, al mismo tiempo que las exigencias de la organización son leves. Por consiguiente, su desempeño es bueno y el nivel de satisfacción alto. Es decir, la experiencia se comporta de la misma manera que la edad. Si el trabajador realiza cambios muy frecuentes de empleo, al comienzo se sentirá muy satisfecho y poco tiempo después muy insatisfecho, lo cual lo puede llevar a cambiar nuevamente de empleo.

- Nivel ocupacional. En cuanto al nivel ocupacional de la persona, mientras más complejo es el trabajo, mayor será el nivel de satisfacción. En otras palabras, los trabajadores menos tecnificados tienden a lograr un nivel más pobre de satisfacción. Esto puede ser una consecuencia del ingreso obtenido por los trabajadores de diferente nivel ocupacional. Como existe una asociación directa entre la cantidad de dinero recibida y el nivel ocupacional dentro del que la persona se ubica, la satisfacción podría deberse al salario más que al nivel de calificación. "En términos generales a un nivel superior corresponde una mayor oportunidad de atender a las necesidades motivadoras (descritas por Herzberg) y más autonomía, interés y responsabilidad" (Dunnette, 1998, p. 266).

- Nivel dentro de la organización. Finalmente, la relación de la satisfacción laboral con el nivel que la persona ocupa dentro de la organización es similar a la que existe con el nivel ocupacional. Si bien el salario puede causar esta relación, hay otros factores que también intervienen. En primer lugar, los profesionales y gerente -muchos de los cuales son también propietarios- gozan de mucho mayor autonomía que los empleados de menor nivel. En segundo lugar, los profesionales y gerentes obtienen refuerzos intrínsecos al realizar su trabajo, lo cual ocurre en menor medida con los trabajadores de nivel inferior. Podemos concluir que la autonomía permite concentrar el esfuerzo en aquellas actividades en las cuales uno encuentra mayor posibilidad de satisfacer las necesidades intrínsecas. Como esta orientación significa -adicionalmenteun mayor ingreso, entonces el nivel organizacional más alto permite lograr una mayor satisfacción. 
- Otros determinantes de la satisfacción laboral. Muchos investigadores se preguntan ¿Qué otras variables relacionadas con el trabajo determinan la satisfacción laboral? Entre los otros factores más relevantes tenemos:

- Intelingencia. La inteligencia en sí no parece ser un factor que repercuta profundamente en la satisfacción del empleado, pero es importante teniendo encuenta el tipo de trabajo que va a ejecutar. En muchas profesiones y ocupaciones hay un límite de inteligencia necesario para un buen rendimiento y satisfacción con el trabajo. En muchas investigaciones se ha demostrado que aquellos cuya inteligencia es demasiado alta para el trabajo que desempeñan, lo juzgan monótono y terminan por sentir tedio y descontento. De manera análoga, cuando alguien desempeña un cargo que requiere una inteligencia superior a la que posee, sentirá frustración si no logra cumplir con las exigencias.

La escolaridad es otro factor que suele relacionarse con la inteligencia. En varios estudios se ha comprobado que las personas con escolaridad más alta experimentan más satisfacción general con su trabajo. Es posible que consigan puestos más interesantes y de mayor autonomía, que ofrecen mejores oportunidades de satisfacer las necesidades de crecimiento.

- Trabajo eventualmente desafiante. Los empleados tienden a preferir trabajos que les den oportunidad de usar sus habilidades y que ofrezcan una variedad de tareas, libertad y retroalimentación sobre cómo se están desempeñando. Los trabajos que tienen muy poco desafío sienten aburrimiento pero, ante un reto moderado, la mayoría de los empleados experimentará placer y satisfacción.

- Recompensas justas. Los empleados quieren sistemas de salarios y políticas de ascensos justos, sin ambigüiedades y acordes con sus expectativas. Cuando el salario se establece con base a las demandas de trabajo, el nivel de habilidad del individuo y los estándares de salario de la comunidad, se favorece la satisfacción. Claro que no todo el mundo busca el dinero; mucha gente acepta con gusto menos dinero a cambio de trabajar en un área preferida, o en un trabajo menos demandante, o de tener mayor discreción en su puesto o de trabajar menos horas, pero la clave en el enlace del salario con la satisfacción no es la cantidad absoluta que uno recibe, sino la percepción de justicia. De igual manera, los empleados buscan políticas y prácticas justas de ascenso. Los individuos que perciben que las decisiones de ascenso se realizan con rectitud y justicia, probablemente experimenten satisfacción.

- Condiciones favorables de trabajo. Los trabajadores se interesan en su ambiente de trabajo tanto para el bienestar personal como para facilitar el hacer un buen trabajo. Los estudios demuestran que los empleados prefieren ambientes físicos que no sean peligrosos o incómodos. La temperatura, la luz, el ruido y otros factores ambientales no deberían estar en el extremo; por ejemplo, tener demasiado calor o muy poca luz. Además, la mayoría de los empleados prefiere trabajar relativamente cerca de la casa, en instalaciones limpias y más o menos modernas, con herramientas y equipo adecuado.

- Colegas que brinden apoyo. La gente obtiene del trabajo mucho más que simplemente dinero o logros tangibles. Para la mayoría de los empleados, el trabajo también cubre la necesidad de interacción social. No es de sorprender, por tanto, que tener compañeros amigables que brinden apoyo lleve a una mayor satisfacción en el trabajo. El comportamiento del jefe es uno de los principales determinantes de la satisfacción del empleado, que se incrementa cuando el supervisor inmediato es comprensivo y amigable, ofrece halagos por el buen desempeño, escucha las opiniones de sus empleados y muestra un interés personal en ellos.

- Personalidad. Holland (2008) expone que la gente con tipos de personalidad congruentes con sus vocaciones escogidas poseerían talentos adecuados y habilidades para cumplir con las demandas de sus trabajos; por tanto, es probable que sean más exitosos en esos trabajos y, debido a este éxito, tengan una mayor probabilidad de lograr una alta satisfacción en su trabajo. 
- Genes. Cuando mucho, 30\% de la satisfacción de un individuo puede explicarse por la herencia genética. Un análisis de los datos relativos a la satisfacción de una muestra de individuos, llevado a cabo durante un período de más de 50 años, encontró que los resultados individuales eran estables con el tiempo, aun cuando estas personas cambiaban de empresa y de ocupación; y la satisfacción de algunas personas, viene determinada genéticamente, se mantiene con el tiempo y se transporta sobre su disposición en el trabajo.

Si usted quiere trabajadores satisfechos "asegúrese de filtrar a los negativos, los inadaptados, los buscadores de problemas, los frustrados, quienes encontrarían poca satisfacción en cualquier aspecto de su trabajo" (Robbins, 1999, p. 153).

\section{EFECTOS DE LA SATISFACCIÓN LABORAL}

Las consecuencias atribuidas al nivel de satisfacción del trabajador en el contexto de la organización son:

- Satisfacción y productividad. Una revisión cuidadosa de la investigación indicó que sí existe una relación positiva entre la satisfacción y la productividad, con correlaciones consistentemente bajas-cercanas a $+0,14$. Esto significa que no más de $2 \%$ de la varianza en la producción puede tomarse en cuenta para la satisfacción del empleado. Sin embargo, la introducción de variables modernas ha mejorado la relación, así tenemos que la relación es más fuerte cuando el comportamiento del empleado no está restringido por factores externos. En el caso de los trabajadores que laboran con máquinas, la productividad estará más influenciada por la velocidad de la misma que por su nivel de satisfacción. El nivel de trabajo también parece ser una variable moderadora importante. Las correlaciones entre la satisfacción y el desempeño son más fuertes para los empleados con niveles más altos.

Una de las razones por las que no se ha obtenido un fuerte apoyo para la tesis de que la satisfacción estimula la productividad es que los estudios se han enfocado en los individuos más que en la organización, y que las mediciones de la productividad a nivel individual no toman en consideración todas las interacciones y complejidades del proceso de trabajo.
- Satisfacción y ausentismo El ausentismo se manifiesta mediante "enfermedades", "problemas familiares", etc. El ausentismo hace que se incurra en mayores gastos médicos, baja de productividad, incremento en costos por personal de reemplazo, etc. Múltiples estudios han sugerido que la insatisfacción conduce al ausentismo. Se ha encontrado en los estudios de una correlación moderada por lo común, menos de $0,40-$.

El hecho de faltar al trabajo puede representar una forma tentativa y breve de alejarse de él. "La causa más frecuente del ausentismo es la baja satisfacción obtenida por la propia realización del trabajo" (Flores, 2000, p 184.)

Otro factor que causa ausentismo son las actividades ajenas a la empresa que ejercen una atracción mayor que el trabajo en sí mismo. Uno puede estar feliz con su trabajo, pero goza más practicando un determinado deporte, $\mathrm{y}$ en ocasiones falta.

Un determinado nivel de ausentismo es ciertamente inevitable debido a enfermedades, condiciones del clima, problemas de transporte, etc. El punto es determinar cuánto ausentismo es aceptable para la organización. Si bien existe una relación directa entre la satisfacción y el ausentismo, no todo el ausentismo es por causa de la insatisfacción laboral.

- Satisfacción y rotación. La satisfacción está relacionada negativamente con la rotación. Los costos de la rotación crecen significativamente a medida que los trabajadores son más calificados o de mayor nivel jerárquico. La rotación demanda mayores gastos en selección de personal y en entrenamiento hasta que la persona alcanza el nivel de competencia requerido. Además, afecta el nivel de productividad y otros aspectos menos obvios, como son las relaciones interpersonales. Si bien existe relación entre satisfacción y rotación, no toda la rotación se debe a la insatisfacción laboral.

- Satisfacción y salud de la persona. La relación entre la salud psicológica de la persona y el nivel de satisfacción con el trabajo no ha sido muy bien investigada. Sin embargo, se asume que hay una correlación positiva. Es necesario determinar hasta qué punto la persona satisfecha con su trabajo lo está también con su vida familiar, con sus amigos, etc.; sólo así se podría determinar si la salud mental es 
una causa o consecuencia de la satisfacción laboral.

Como lo señala Johns (1988), diferentes estudios han mostrado que los trabajadores que describen su trabajo como insatisfactorio tienden a sufrir múltiples síntomas y enfermedades físicas. La asociación entre salud y satisfacción no indica que una cause la otra. Si bien no existen muchas evidencias que respalden esta postura, es necesario tener en cuenta que una persona que se encuentra satisfecha en su trabajo tendrá actitudes más positivas hacia la vida, lo que influirá en el equilibrio de su salud física y psicológica.

\section{Investigaciones sobre la satisfacción laboral}

- En el extranjero. La serie de investigaciones conocida como los estudios de Hawotharne, de la Wester Electric Company de Chicago en 1927, bajo la dirección de Elton Mayo, de Harvard Bussines School, reconocieron la importancia de la supervisión sobre las actitudes de los trabajadores.

Mediante entrevistas establecieron cuestionarios muy estructurados sobre qué le agradaba o disgustaba a cada empleado en materia del ambiente físico del trabajo, supervisión y aspectos de la tarea. Los resultados de la entrevista se emplearon para mejorar las relaciones humanas. El estudio demostró que la satisfacción o insatisfacción tenía poca relación con el ambiente físico del trabajo. La satisfacción o insatisfacción dependía de las actitudes del grupo.

En una reciente encuesta, Gallup consideró a los estadounidenses de diversas partes de la nación para conocer sus actitudes hacia sus trabajos y sus lugares de trabajo. En una escala del 1 al 5 en la que 5 representa “extremadamente satisfecho", 71\% de los encuestados calificaron su nivel de satisfacción con su lugar de empleo con 4 o 5 , mientras sólo 9\% lo calificó con 1 o 2 . Consideraron los siguientes factores críticos para su satisfacción y desempeño en el trabajo: la oportunidad de hacer lo que ellos hacen mejor; lograr que sus opiniones cuenten, conseguir la oportunidad de aprender y crecer. De los que respondieron, 62 indicaron que en los siete días anteriores habían recibido el reconocimiento o la aprobación por un buen trabajo; $84 \%$ dijo que había tenido la oportunidad de trabajar para aprender y crecer durante el año anterior. Estos resultados son aplicables a lo largo del tiempo como también a lo largo de las fronteras nacionales. Sin importar los estudios que se deseen observar, cuando a los trabajadores se les pregunta si están satisfechos con sus trabajos, los resultados tienden a ser muy similares: entre 70 y $80 \%$ informan que sí se sienten satisfechos en sus puestos. Estos números tienden a variar con la edad -conforme más maduros son los trabajadores más alta es la satisfacción-. Pero aún la gente joven (menores de 25 años) informan niveles de satisfacción excesiva de $70 \%$. Además, estos resultados son generalmente aplicables a otros países desarrollados. Por ejemplo, estudios comparables entre trabajadores en Canadá, Gran Bretaña, Suiza, Alemania, Francia y México indican más resultados positivos que negativos.

Muñoz, A. (1989), de la Universidad Complutense de Madrid, obtiene resultados que lo llevan a suponer que, si bien las personas pueden no estar tan insatisfechas en su trabajo como suponíamos, tampoco están satisfechas. Se confirma la existencia de un factor general de satisfacción, insatisfacción laboral y la multidimensionalidad de esta variable, aunque con un contenido diferente para sujetos satisfechos e insatisfechos. Se encontró una relación positiva entre satisfacción en el trabajo y gusto con la tarea, motivación para el trabajo, moral laboral, proceso seguido por la motivación y voluntad de volver a la empresa, y nula o no significativa con las demás variables laborales. Por ultimo, encontró que no existe relación significativa entre satisfacción en el trabajo y las variables demográficas y profesionales.

Otro estudio es realizado por el Ministerio de Trabajo y Asuntos Sociales de España (2002). Se aplicó una encuesta en donde confirman que los trabajadores españoles manifiestan un nivel medio de satisfacción en el trabajo, al situarse en 6,79 puntos, en una escala de 0 a 10 , en la que cero es ninguna satisfacción y 10 muy satisfecho. Los datos anteriores forman parte de los resultados obtenidos por la tercera Encuesta de Calidad de Vida en el Trabajo, que el Ministerio de Trabajo y Asuntos Sociales realizó en 2001. El 89,7\% de los entrevistados respondieron que estaban satisfechos o muy satisfechos con su trabajo, 
lo que significa una mejora con respecto a la valoración sobre el mismo tema de años anteriores: $89,1 \%$ en 1999 y un $86,7 \%$ en el 2000. Las principales fuentes de gratificación laborales que mencionan los entrevistados son: el gusto por la realización de su trabajo $(24,5 \%)$, el compañerismo $(11,8 \%)$, el sueldo $(7,7 \%)$, el buen horario $(7,5 \%)$ y el desarrollo personal $(7,4 \%)$.

- En nuestro medio. En el Perú se han sustentado planteamientos relacionado con factores intrínsecos del trabajador, así tenemos los trabajos de León, F. (1976) y León y Pejerrey G. (1978).

En una investigación sobre factores considerados por empleados de oficina y trabajadores manuales al "ofrecerles un puesto", pusieron a comprobación la hipótesis que los empleados de oficina peruanos, en comparación con los trabajadores manuales, son estimulados más poderosamente por los aspectos intrínsecos del trabajo (propiedades de la actividad laboral en sí, particularmente aquellas relacionadas con el crecimiento psicológico en el trabajo) y menos poderosamente por los extrínsecos (características del entorno o contexto del trabajo).

De los resultados obtuvieron que tanto en los trabajadores manuales como entre los empleados de oficina, los factores intrínsecos mostraron ser causas más poderosas de satisfacción-insatisfacción que los factores extrínsecos.

León, F., Loli, A. y Montalvo, A. (1975), así como Álvarez, D. (1994), encontraron una alta relación de la satisfacción laboral con las expectativas de poner en juego las habilidades personales en el puesto de trabajo, asimismo reportaron mayor satisfacción laboral a nivel de gerentes y profesionales frente a los de menor jerarquía de puesto; también la variable tiempo de servicios presenta una relación directa decreciendo ésta a partir de los 10 años de servicio. En relación a la edad, no muestra un patrón uniforme y los aspectos intrínsecos como logro y responsabilidad fueron de mayor puntuación, mientras que el de remuneración como factor extrínseco obtuvo menor puntuación.

Torres (1987) realizó una investigación sobre la satisfacción laboral que experimenta la enferme- ra general durante el ejercicio de su profesión en los hospitales E. Rebagliati y Dos de Mayo.

Se llegó a la conclusión que el $65 \%$ de las enfermeras experimentan insatisfacción laboral, el 35\% experimentan satisfacción. Los factores que determinan la insatisfacción laboral son los extrínsecos: organización, salario y ambiente físico. Al comparar la satisfacción laboral entre las enfermeras de ambas instituciones, se obtuvo que en el hospital E. Rebagliati, el $60 \%$ de las enfermeras experimentan insatisfacción laboral, mientras que en el hospital Dos de Mayo, el 90\% de las enfermeras experimentan insatisfacción laboral al realizar su ejercicio profesional

Velásquez (1982), en un estudio realizado a empleados bancarios que pertenecen a diferentes puestos de trabajo, encontró que la satisfacción laboral está en función no sólo de lo que gana por su situación laboral, sino también por el lugar donde se encuentra la pesona respecto a su nivel de aspiraciones. Afirma también que cuando el ambiente ofrece pocas posibilidades para la satisfacción de las necesidades, este tipo de personas serán las menos felices. También halló que ambos géneros tenían actitudes parecidas frente al puesto de trabajo y hacia la empresa, afirmando que a mayor grado de instrucción alcanzado por un empleado, logra puntajes más altos y por ende logran mayor satisfacción y, a menor grado de instrucción, tienden a tener puntajes bajos.

Izquierdo (1983), en un estudio realizado en el Banco Central de Reserva del Perú acerca de las actitudes de un grupo de trabajadores de diferente nivel jerárquico, afirma que la satisfacción laboral puede contribuir eficazmente al éxito y desarrollo personales pero, para que los trabajadores se sientan satisfechos, se requiere un alto grado de comprensión e integración de ellos con la empresa. Reportó que existe relación entre la actitud positiva y el cargo jerárquico que se desempeña. No hay relación entre las actitudes positivas con respecto a la edad cronológica, existe independencia entre estas dos variables. No hay relación de la actitud positiva con respecto al género pero sí existe relación con respecto al grado de instrucción.

Palma (1999), reportó en una muestra de 952 trabajadores de cinco grupos ocupacionales, 
un nivel de satisfacción promedio en trabajadores dependientes en Lima Metropolitana, siendo los factores intrínsecos los de mayor satisfacción. Las diferencias por género favorecen a la mujer con un mejor nivel de satisfacción laboral; así también ocurre con los trabajadores que atienden directamente al público, siendo la remuneración un elemento directamente asociado al grado de satisfacción.

\section{MÉTODOS Y RESULTADOS}

El presente estudio será una base importante para estudios posteriores, pero también es necesario considerar que la Escala de Satisfacción Laboral (SL-SPC) es el primer instrumento estandarizado en el Perú que cuenta con baremos adecuados para brindar resultados válidos en el área organizacional.

La Facultad de Ciencias Administrativas pertenece a la Universidad Nacional Mayor de San Marcos y cuenta con personal docente (permanente y contratado), personal administrativo (permanente, designado y contratado) y alumnos.

Para cumplir con el objetivo de esta investigación se ha tomado la prueba solo al personal administrativo.

Materiales: Escala de Satisfacción Laboral (SLSPC)

La Escala de Satisfacción Laboral (SL-SPC) fue elaborada por Sonia Palma Carrillo (magíster de la Universidad Ricardo Palma), en el año 1999, prueba elaborada utilizando el método likert.

Esta escala fue validada y estandarizada en Lima Metropolitana con la ayuda de los estudiantes de postgrado de la Universidad Ricardo Palma, utilizando una población conformada por 838.591 trabajadores en Lima Metropolitana (INEI, 1993) de la cual se extrajo una muestra accesible (8.386) equivalente a 952 empleados.

- Definición de las escalas de la prueba. La escala cuenta con 36 proposiciones asociados a siete factores que se definen como siguen:

- Factor I: Condiciones físicas y/o materiales (5 ítems). Los elementos materiales o de infraestructura donde se desenvuelve la labor cotidiana de trabajo y se constituye como facilitador de la misma.
- Factor II: Beneficios laborales y/o remunerativos (4 ítems). El grado de complacencia en relación con el incentivo económico regular o adicional como pago por la labor que se realiza.

- Factor III: Políticas administrativas (5 ítems). El grado de acuerdo frente a los lineamientos o normas institucionales dirigidas a regular la relación laboral y asociadas directamente con el trabajador.

- Factor IV: Relaciones sociales (4 ítems). El grado de complacencia frente a la interrelación con otros miembros de la organización con quien se comparte las actividades laborales cotidianas.

- Factor V: Desarrollo personal (6 ítems). Oportunidad que tiene el trabajador de realizar actividades significativas a su autorrealización.

- Factor VI: Desempeño de tareas ( 6 ítems). La valoración con la que se asocia el trabajador con sus tareas cotidianas en la entidad donde labora.

- Factor VII: Relación con la autoridad (6 ítems). La apreciación valorativa que realiza el trabajador de su relación con el jefe directo y respecto a sus actividades cotidianas.

- Participantes. Este estudio contó con la participación de:

- Personal permanente (nombrado): 12 personas

- Personal designado (planilla): 7 personas

- Personal contratado (CAS) : 45 personas

Se ha tomado el íntegro del personal permanente y designado, y a 30 personas del personal contratado, ya que algunas de ellas fueron cambiadas y/o se encontraban de permiso y/o de vacaciones sin distinción de sexo, cargo o posición en la Facultad.

\section{ANÁLISIS Y DISCUSIÓN}

El nivel de la satisfacción laboral del personal administrativo de la Facultad es regular. En primer lugar es necesario recalcar que la satisfacción laboral de los trabajadores de la Facultad de Ciencias Administrativas está determinada por los factores intrínsecos del trabajo más que por los extrínsecos, tal como lo afirma Herzberg (2007) en sus estudios. En este sentido, cabe mencionar 
que los mayores puntajes, es decir, lo que favorece el nivel de satisfacción laboral en el personal administrativo de la Facultad, son el desempeño de tareas, el desarrollo personal y, en menor proporción, lo que lleva a una insatisfacción laboral son las condiciones físicas y beneficios laborales y/o remunerativos. Es necesario citar a Velásquez (2002) quien afirma que la satisfacción laboral es multidimensional, ya que es posible que el personal administrativo se sienta satisfecho en ciertos aspectos de su trabajo y, al mismo tiempo, descontento con otros.

Los resultados según la variable categoría ocupacional son los siguientes: a nivel general, los puntajes del personal administrativo permanente y contratado son comparativamente iguales, es decir, no existen diferencias significativas y, por lo tanto, las actitudes que tienen frente a la satisfacción laboral son similares.

Sin embargo, en los factores que conforman la satisfacción laboral, se aprecia que existen diferencias significativas en el factor de relaciones sociales. El personal administrativo contratado percibe que sus compañeros no permiten que su labor sea más placentera y, por lo tanto, se sienten descontentos con este factor.

Es necesario observar que, de acuerdo a los niveles de diagnóstico de la satisfacción laboral, también se encontraron diferencias significativas en este factor, pues es el personal administrativo contratado el que tiene un mayor porcentaje de evaluados en los niveles de parcial y alta insatisfacción laboral, a comparación del personal administrativo permanente quienes muestran altos puntajes en estos niveles de diagnóstico y, por lo tanto, tienen mayores índices de satisfacción laboral a comparación de los contratados. No se han encontrado investigaciones anteriores que respalden este resultado, pero se asume que tiene que ver con la estructura característica de la Facultad (ya que el 66 $\%$ del personal administrativo permanente esta con cargo de Jefatura) y con otras variables que pueden estar interviniendo, más externas que internas.

También se observan diferencias muy significativas de acuerdo al factor de relación con la autoridad, es decir, son los contratados los que muestran insatisfacción con respecto a la apreciación valorativa frente a su jefe inmediato. Esto puede deberse al tipo de manejo y de supervisión impartido por los jefes y directores inmediatos en la Facultad.
Asimismo, se aprecian diferencias significativas con respecto al factor de políticas administrativas. Aquí nuevamente son los contratados quienes muestran insatisfacción frente a los lineamientos o normas institucionales impartidas por la Facultad. Según fuentes consultadas en la Facultad esto puede deberse a que se encuentran menos vinculados con las acciones que la Facultad realiza para cumplir con los objetivos que se propone, es decir, físicamente son los más alejados a las Direcciones o Decanato, y la forma de retroalimentación es insuficiente pues no permite mantener informado a este sector sobre las metas logradas o sobre los problemas que se presentan, ya sea con el personal docente, administrativo o alumnos.

Finalmente, se observan diferencias significativas con respecto a los beneficios laborales y/o remunerativos; es decir, tanto permanentes como contratados se muestran insatisfechos con respecto al incentivo económico, pero en mayor proporción son los contratados los que experimentan una mayor insatisfacción laboral. Es importante apreciar que ello puede estar influido porque sus sueldos no son competitivos en el mercado, a comparación de el de los permanentes, quienes están dentro del promedio, lo cual puede generar insatisfacción en los contratados a la hora de compararse con otros ambientes laborales del mismo rubro.

Es necesario recalcar que son los factores extrínsecos del trabajo, tales como relaciones sociales, relación con la autoridad, políticas administrativas y beneficios laborales, los que generan mayor insatisfacción a los contratados de la Facultad a comparación de los administrativos permanentes, quienes sí manifiestan muy poca satisfacción hacia estos factores.

\section{CONCLUSIONES}

1. El personal administrativo permanente y el contratado presentan actitudes similares de satisfacción laboral y, de acuerdo a la categoría de diagnóstico, presentan un nivel regular de satisfacción laboral.

2. Los factores que favorecen la satisfacción laboral son el desempeño de tareas, el desarrollo personal y las relaciones sociales, siendo las que menos la favorecen los factores de condiciones físicas y remuneración.

3. El personal administrativo contratado tiene un mayor nivel de insatisfacción laboral en 
aspectos tales como relación con la autoridad, políticas administrativas y relaciones sociales, a diferencia de los permanentes quienes sí muestran satisfacción hacia estos factores. Con respecto al factor de beneficios laborales y/o remunerativos, tanto contratados como permanentes se muestran insatisfechos con respecto al incentivo económico, pero en mayor proporción son los contratados los que experimentan una mayor insatisfacción laboral.

4. Se recomienda realizar este tipo de diagnósticos por lo menos cada dos años, de tal forma que se puedan apreciar los cambios que se establezcan para bien del personal administrativo en general y de la Facultad.

\section{LITERATURA CITADA}

Álvarez, M. (2005). El liderazgo y la calidad total. Editorial Escuela Española, España.

Beckhard, R. (2003). Desarrollo organizacional: estrategias y modelos. Centro Regional de Ayuda Técnica, México.
Collins, D. (2006). "Nuevos paradigmas para el cambio: teorías de la organización y las organizaciones de teorías". En: Journal of Organizational Change Management. Emerald Group Publishing. Reino Unido, Vol. 9 № 4.

Florez, J. (2002). El comportamiento humano en las organizaciones. Universidad del Pacífico, Perú.

Hanna, D. (2002). Diseño de organizaciones para la excelencia en el desempeño. Addison Wesley Iberoamericana, Estados Unidos.

Munch, L. (2002). Más allá de la excelencia y la calidad total. Ed. Trillas, México.

Palma, S. (2003). "Trabajo y salud laboral". En: Revista Pirámide. Facultad de Psicología de la Universidad Ricardo Palma. Lima, Año 4, №1.

Rodríguez, D. (2007). Diagnóstico organizacional. Alfaomega, México.

Toro, F. (2001). El clima organizacional. Perfil de las empresas colombianas. Cincel, Colombia. 